\title{
UNION ASTRONOMIQUE INTERNATIONALE
}

\section{STATUTS}

\section{BUT}

1. L'Union Astronomique Internationale (ci-après denommée Union) est une organisation internationale non-gouvernementale. Son but est de promouvoir la science de l'astronomie sous tous ses aspects.

\section{DOMICILE ET RELATIONS INTERNATIONALES}

2. L'Union a son siège légal à Paris.

3. L'Union adhère il et coopere avec I'ensemble des organisations scientifiques par le biais de l'ICSU: Conseil International pour la Science. EIle apporte son soutien et met en æuvre les politiques de Liberté, Responsabilité et Ethique dans la Conduite de la Science, telles que définies par l'ICSU.

\section{COMPOSITION DE L'UNION}

4. L'Union se compose :

4.a. de Membres Nationaux (organisations adhérentes),

4.b. de Membres Individuels (personnes adhérentes).

\section{LES MEMBRES NATIONAUX}

5. Une organisation représentant une communauté nationale astronomique professionnelle souhaitant promouvoir sa participation au niveau de I'astronomie internationale et soutenant le but de l'Union, peut adhérer il cette derniere en qualité de Membre National. Exceptionnellement, un Membre National peut representer la comrnunaute d'un territoire composé de plusieurs pays, sous réserve qu'aucune partie de cette communauté ne soit representée par un autre Membre National.

6. Une organisation souhaitant joindre l'Union en qualité de Membre National alors qu'elle développe I'astronomie professionnelle au sein de la communauté qu'elle represente peut le faire sur une base intérimaire, dans les mêmes conditions que décrites ci-dessus et ce pour une période maxirnum de neuf ans. Aprés cette période, elle devient Membre National permanent ou démissionne de l'Union.

7. Un Membre National, qu'il soit permanent ou intérimaire, est admis au sein de l'Union par l' Assemblée Générale. Il peut démissionner de I'Union en en informant par écrit le Secrétaire Général.

8. Un Membre National peut être:

8.a. soit l'organisation par laquelle les scientifiques du pays ou du territoire correspondant adhérent a l'ICSU,

8.b. soit une société savante nationale appropriée ou Comite National pour l'Astronomie,

8.c. soit une institution d'enseignement de haut niveau appropriée. 
9. L'adhesion d'un Membre National est suspendue s'il n'a pas payé sa contribution durant cinq ans. Cette suspension est levée, aprés accord du Comite Exécutif, lorsque les arrérages ont été payés. Aprés cinq années de suspension d'un Membre National, le Comité Exécutif peut recommander el rAssemblée Générale de mettre fin el son adhésion.

10. Un Membre National est admis au sein de l'Union dans l'une des catégories définies dans le Réglement.

\section{LES MEMBRES INDIVIDUELS}

11. Un scientifique professionnel æuvrant dans toute branche de I'astronomie peut etre admis au sein de l'Union en qualité de Membre Individuel par le Comite Exécutif. Tout Membre lndividuel peut démissionner de rUnion en en informant son Sécretaire Général par écrit.

\section{DIRECTION}

12. Les instances de direction sont;

12.a. L'Assemblée Générale;

12.b. Le Comité Exécutif; et

12.c. Les Administrateurs.

\section{L'ASSEMBLEE GENERALE}

13. L'Assemblée Générale est constituée des Membres Nationaux et des Membres lndividuels. L'Assemblee Generale décide de la politique générale de l'Union.

13.a. L'Assemblée Générale approuve les Statuts de l'Union, ainsi que toute modification de ces statuts.

13.b. L'Assemblée Générale approuve un Réglement qui précise les modalités d'application des Statuts.

13.c. L'Assemblée Générale élit un Comité Exécutif chargé de mettre en æuvre ses décisions et de conduire les affaires de l'Union entre les réunions successives des Assemblées Générales ordinaires. Le Comité Exécutif rend compte de sa gestion auprés de l'Assemblée Générale.

13.d. L'Assemblée Générale nomme un Comité des Finances composé d'un représentant. de chacun des Membres Nationaux ayant le droit de voter conformément à l'article 14.a., lequel est chargé de la conseiller dans l'approbation du budget et des comptes de l'Union. L'Assemblée Générale nomme aussi un Sous-Comité des Finances pour conseiller en son nom le Comité Exécutif sur les affaires budgétaires entre les Assemblées Générales.

13.e. L'Assemblée Générale nomme un Comité Spécial des Nominations pour la préparation d'une liste appropriée de candidats pour élection au Comité Exécutif entrant.

13.f. L'Assemblée Générale nomme un Comité des Nominations chargé de faire des recommandations au Comité Exécutif en ce qui concerne l'admission des Membres Individuels. 
14. Les votes lors de l'Assemblé Générale sont exercés par les Membres Nationaux. Chaque Membre National désigne un représentant pom voter en son nom.

14.a. Sur les questions engageant le budget de l'Union, le nombre de voix de chaque Membre National est égal à l'indice de sa catégorie, telle que définie à l'article 10., augmenté d'une unité. Les Membres Nationaux intérimaires ou qui n'ont pas payé leurs cotisations durant les années précédant celle de l'Assemblée Générale ne peuvent pas participer aux votes.

14.b. Sur les questions concernant l'administration de l'Union sans implication budgétaire, chaque Membre National dispose d'une voix, dans les mêmes conditions quant au payment des cotisations qu'en 14.a.

14.c. Les Membres Nationaux peuvent voter par correspondance sur les questions concernant l'ordre du jour de l'Assemblée Générale.

14.d. Un scrutin n'est valable que si au moins deux tiers des Membres Nationaux disposant du droit de vote en vertu de l'article 14.a. y prennent part.

15. Les décisions prises par l'Assemblée Générale le sont el. la majorite absolue des suffrages. Cependant, une décision de modification des Statuts n'est valable que si elle a réuni la majorité des deux tiers des voix de tous les Membres Nationaux qui disposent du droit de vote en vertu de l'article 14.a. En cas de partage égal des voix, le résultat est détermine par le Président.

16. Une modification des Statuts ou du Réglement ne peut être soumise ei l'Assemblée Générale que si une proposition en ce sens a été soumise aux Membres Nationaux et figure, en tant que telle, à l'ordre du jour de l'Assemblée Générale, conformément aux régles et dates limites indiquées dans le Réglement.

\section{LE COMITE EXECUTIF}

17. Le Comité Exécutif se compose du Président de l'Union, du Président-Elect, de six Vice-Présidents, du Secrétaire Général et du Secrétaire Général Adjoint élus par l'Assemblée Générale sur proposition du Comité Spécial des Nominations.

\section{LES ADMINISTRA TEURS}

18. Les Administrateurs de l'Union sont le Président, le Secrétaire Général, le PrésidentElect et le Secrétaire Général Adjoint. Les Administrateurs décident de la politique à court terme, en accord avec la police générale de l'Union telle qu'elle a été décidée par l'Assemblée Générale et mise en æuvre par le Comité Exécutif.

\section{LES DIVISIONS SCIENTIFIQUES}

19. Afin de promouvoir efficacement l'avancement des diverses branches de l'astronomie, le travail scientifique de l'Union est organisé au sein de ses Divisions Scientifiques. Chaque Division couvre une branche large et bien définie de la science de l'astronomie, ou bien s'occupe de sujets intemationaux de nature interdisciplinaire. Dans la mesure du possible, les Divisions comportent une fraction comparable de Membres Individuels de l'Union.

20. Les Divisions sont créées ou dissoutes par l'Assemblée Générale sur recommandation du Comité Exécutif. Les activités des Divisions sont organisées par un Comité d'Organisation présidé par un Président de Division. Le Président et un VicePrésident sont élus par l'Assemblée Générale sur proposition du Comité Exécutif et sont membres du Comité d'Organisation ex officio. 


\section{LES COMMISSIONS SCIENTIFIQUES}

21. Au sein des Divisions, les activités scientifiques de thémes ciblés entrant dans le domaine de la Division, sont organisées par des Commissions Scientifiques. Dans certains cas, une Commission peut couvrir des sujets communs à deux ou plusieurs Divisions et devient alors une Commission de ces Divisions.

22. Les Commissions sont créées ou dissoutes par le Comité Exécutif, sur recommandation dul des Comité(s) d'Organisation de la/des Division(s) souhaitant les créer ou les dissoudre. Les activités d'une Commission sont organisées par un Comité d'Organisation préside par un Président de Commisison. Le Président et un VicePrésident sont nommés par le(s) Comité(s) d'Organisation de la/des Division(s) correspondante(s) sur proposition du Comité d'Organisation de la Commission.

\section{BUDGET ET COTISATIONS}

23.a. Pour toute Assemblée Générale ordinaire, le Comité Exécutif prépare un projet de budget pour la période el courir jusqu'à l'Assemblée Générale ordinaire suivante, ainsi que les comptes de l'Union pour la période précédente. Il les soumet, assortis des commentaires du Sous-Comité des Finances, au Comité des Finances pour examen avant leur mise au vote de l'Assemblée Générale.

23.b. Le Comité des Finances scrute les comptes de l'Union et vérifie que les dépenses engagées l'ont été conformément aux décisions de la précédente réunion de l'Assemblée Générale, telles qu'elles ont été interprétées par le Comité Exécutif. Il s'assure aussi que le budget proposé permet la mise en æuvre de la politique de l'Assemblée Générale. Il présente des rapports sur ces questions à l'Assemblée Générale avant toute décision relative à l'approbation des comptes et du budget.

23.c. Le montant de l'unité de contribution est entériné par l'Assemblée Générale comme partie intégrante du processus d'approbation du budget.

23.d. Chaque Membre National verse annuellement un nombre d'unités de cotisation correspondant à sa catégorie. Le nombre d'unités de cotisation de chaque catégorie est fixé par le Réglement.

23.e. Les Membres Nationaux intérimaires paient une demi-unité de contribution.

23.f. Le paiement des cotisations est a la charge des Membres Nationaux. Les Membres Nationaux n'ont aucune autre responsabilité financiére envers l'Union.

\section{DEVOLUTION DE L'AUTORITE EN CAS DE FORCE MAJEURE}

24. $\mathrm{Si}$, par suite d'evénements indépendants de la volonté de l'Union, les circonstances sont telles qu'elles rendent impossible le respect des clauses du Statut et du Réglement de l'Union, le Comité Exécutif et les Administrateurs prendront toutes dispositions qu'ils jugeront nécessaires pour la continuation du fonctionnement de l'Union. Ces dispositions devront être communiquées des que possible il tous les Membres Nationaux, jusqu'à ce qu'une Assemblée Générale ordinaire ou extraordinaire puisse être réunie.

L'autorité est dévolue dans l'ordre suivant: au Comité Exécutif, réuni ou par correspondance, au Président de l'Union, au Secrétaire Général ou, à défaut de cette possibilité ou de la disponibilité des autorités ci-dessus mentionnées, à l'un des Vice-Présidents. 


\section{DISSOLUTION DE L'UNION}

25. Toute décision de dissolution de l'Union n'est valide que si elle est prise par décision de l'Assemblée Générale ayant réuni les trois-quarts des Members Nationaux en droit de voter selon l'article 14.a. Une telle décision est assortie d'une procédure de réglement des dettes et de distribution des avoirs de l'Union.

\section{CLAUSES FINALES}

25. Ces Statuts entrent en vigueur le 15 juiliet 2003.

26. Les présents Statuts sont publiés en versions francaise et anglaise. En cas d'incertitude, la version francaise fait seule autorité. 